\title{
Hepatotoxicity caused by montelukast in a paediatric patient
}

\author{
Dariusz M. Lebensztejn ${ }^{1}$, Anna Bobrus-Chociej ${ }^{1}$, Monika Kłusek ${ }^{1}$, Miroslawa Uscinowicz ${ }^{1}$, Joanna Lotowska ${ }^{2}$, \\ Maria Sobaniec-Lotowska², Maciej Kaczmarski ${ }^{1}$ \\ ${ }^{1}$ Department of Paediatrics, Gastroenterology and Allergology, Medical University of Bialystok, Poland \\ ${ }^{2}$ Department of Clinical Pathomorphology, Medical University of Bialystok, Poland
}

Prz Gastroenterol 2014; 9 (2): 121-123

DOI: $10.5114 / p g .2014 .42509$

Key words: montelukast, hepatotoxicity, drug-induced liver disease, children.

Address for correspondence: Prof. Dariusz M. Lebensztejn, Department of Paediatrics, Gastroenterology and Allergology, Medical University of Bialystok, 17 Waszyngtona St, 15-247 Bialystok, Poland, phone: +48 8574505 39, e-mail: lebensztejn@hoga.pl

\begin{abstract}
Montelukast is a selective and competitive cysteinyl leukotriene receptor antagonist (CystLTRA) which is increasingly used for the treatment of allergic asthma. Recently, hepatotoxicity has been reported with this drug in adult patients, but only one letter to the editor has reported a case of probable montelukast-induced hepatotoxicity in a child. We present a case of a 3.5-year-old boy, receiving treatment with montelukast, who developed hepatocellular injury. The exclusion of other causes of increased activity of aminotransferases (viral, metabolic, autoimmune), improvement after dechallenge, the morphological findings and previous reports of comparable cases support the diagnosis of montelukast-induced liver injury in this boy. Physicians should strictly analyse indications for this drug and be aware of potential drug-induced liver disease caused by this agent. Therefore, the periodical assessment of aminotransferases should be recommended during treatment with this leukotriene modifier.
\end{abstract}

\section{Introduction}

Drug-induced hepatotoxicity, i.e. the toxic effect of a chemical substance on the liver, is a term describing pathologic changes occurring in this organ as a result of prophylactic or therapeutic use of a pharmacological agent. The diagnosis of drug-induced liver disease (DILI) includes the time of onset, clinical features, the time and course of recovery, specific risk factors, the exclusion of other diagnosis and previous reports on the hepatotoxicity of the implicated agent. The diag nosis can be strengthened by rechallenge and, in some cases, liver biopsy [1]. For most drugs, the risk of liver injury is estimated to be 1-10/100,000 exposed medical inpatients [2]. Liver damage due to the use of drugs is considerably less frequent in children than in adults, but it is still a significant reason for acute liver failure [3].

The aim of the paper is to present a case of a child, receiving treatment with montelukast, who developed hepatocellular injury.

\section{Case report}

Our report presents data concerning a non-obese 3.5-year-old boy. There was no prior family history of liver disease. The patient had a history of suspected allergic asthma (prick-test reactivity with house dust mite/Dermatophagoides pteronyssinus and farinae/ pollen allergens), and for that reason he had been prescribed montelukast at a $5 \mathrm{mg}$ daily dose for the last 5 months before being admitted to our hospital.

He was admitted to the Department of Paediatric Infectious Diseases of the Medical University of Medical University of Bialystok on 6 March 2010 with hypertransaminasaemia, which was diagnosed in the outpatient clinic (diagnostics of abdominal pain). The activity of liver enzymes was: alanine aminotransferase (ALT) 1197 IU/I (reference limits 10-40 IU/l), asparagine aminotransferase (AST) - $490 \mathrm{IU} / \mathrm{l}$ (reference limits 10-40 IU/l), alkaline phosphatase (APT) - $305 \mathrm{IU} / \mathrm{I}$ (reference limits 110-350 IU/I) and $\gamma$-glutamyl transferase (GGT) - $78 \mathrm{IU} / \mathrm{l}$ (reference limits 1-40 IU/l). The bilirubin level was $0.82 \mathrm{mg} / \mathrm{dl}$ (reference limits $0.1-1.1 \mathrm{mg} / \mathrm{dl}$ ).

Viral hepatitis - HBV (HBsAg negative), HCV (antiHCV negative), HAV (anti-HAV IgM negative), CMV (anti-CMV IgM negative), HSV (anti-HSV IgM negative), infectious mononucleosis as well as toxoplasmosis were excluded. Treatment with hepatoprotective drugs 
(essential phospholipids and silymarin) was started and, after consultation witha hepatologist, who took into consideration DILI, the montelukast therapy was stopped on 10 March 2010.

The patient was transferred to the Department of Paediatrics, Gastroenterology and Allergology of the Medical University of Bialystok on 12 March 2010 for further evaluation of increased activity of serum ALT.

On admission, the boy was in generally good condition. Pruritis was the only symptom. No abnormalities except skin rash and hepatomegaly were found in the physical examination. Increased activity of transaminases (ALT - $894 \mathrm{IU} / \mathrm{I}, \mathrm{AST}-510 \mathrm{IU} / \mathrm{l})$ and GGT (78 IU/l) were found (15 March 2010). The level of total and direct bilirubin $(0.74 \mathrm{mg} / \mathrm{dl}$ and $0.21 \mathrm{mg} / \mathrm{dl}$, respectively) was normal. Prothrombin time (13.2 s) prothrombin index (92\%), albumin (4.6 g/l), leucocytes count $\left(6.67 \times 10^{3} / \mu \mathrm{l}\right)$ and eosinophils count $(200 / \mu \mathrm{l}-3.6 \%)$ were also normal. We found slightly increased concentration of total immunoglobulin E $(42 \mathrm{lU} / \mathrm{ml}$, reference limits up to $33 \mathrm{IU} / \mathrm{l})$. Wilson disease, cystic fibrosis, autoimmune hepatitis, $\alpha 1$-antitrypsin deficiency and celiac disease were excluded. We did not find HBV DNA or HCV RNA in serum, nor abnormal results in GC-MS and Tandem mass spectroscopy examination.

Hyperechogenicity of liver parenchyma was observed in ultrasound examination. Five days later (19 March 2010) we did not observe a significant decrease of ALT activity (903 IU/l), although the skin rash and pruritis were absent. Therefore, liver biopsy was performed (20 March 2010) to elucidate the still unclear cause of hypertransaminasaemia. Histopathological ex-

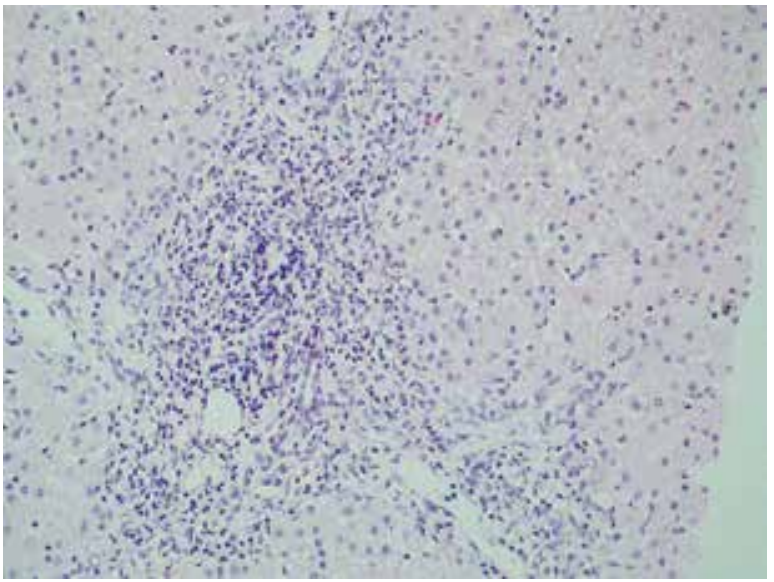

Figure 1. Histopathological examination of liver bioptate revealed that the portal tract exhibited a dense lymphohistiocytic infiltration focally with an admixture of eosinophils. The inflammatory infiltrate focally exceeded the limiting plate of the liver lobule $(H+E$ stain, $200 x)$ amination of liver bioptate revealed that the portal tract exhibited a dense lymphohistiocytic infiltration focally with an admixture of eosinophils. The inflammatory infiltrate focally exceeded the limiting plate of the liver lobule (Figure 1).

The activity of ALT $(758 \mathrm{IU} / \mathrm{l})$ and AST (380 IU/I) showed a tendency to normalisation (22 March 2010). Two months after montelukast termination the ALT and GGT activity decreased to $116 \mathrm{IU} / \mathrm{I}$ and $29 \mathrm{IU} / \mathrm{l}$, respectively, and a month later the activity of ALT became normal (37 IU/I). The boy was still a patient of the Outpatient Gastroenterology Clinic, and 12 months after montelukast termination the activity of ALT was at the normal level.

\section{Discussion}

The clinical picture of drug-induced liver injury is variable, ranging from asymptomatic mild elevation of liver enzymes (ALT and/or GGT) and cholestasis to acute liver failure. Liver injury is classified into 3 types: hepatocellular, cholestatic and mixed [3]. In the present case, ALT activity was more than 20 times above upper limits of normal (ULN) and the ratio of serum activity of ALT to alkaline phosphatase (both expressed as multiple of the ULN) was $>5$, being classified as hepatocellular injury type.

This boy was exposed to the potential hepatotoxic drug: montelukast. Montelukast is a selective and competitive cysteinylleukotriene receptor antagonist (CystLTRA) which is increasingly used for the treatment of allergic asthma. Recently, hepatotoxicity has been reported with this drug in adult patients [4-9], but only one letter to the editor has reported a case of probable montelukast-induced hepatotoxicity in a child [10].

The rare incidence of montelukast-induced liver injury and the long latency in the appearance of liver necrosis (in this case 5 months) suggest a metabolic idiosyncrasy that may be due to pharmacogenetic factors and may be triggered by environmental factors.

We did not perform rechallenge and we did not use semiqualitative clinical scoring systems to increase the specificity of the diagnosis of DILI, such as RUCAM/ CIOMS. According to Murray et al. [3], their routine use is not recommended in paediatric patients because the specificity of some assessed parameters is considerably lower in children (ALP activity, alcohol abuse, pregnancy or hypotension of cardiac origin). Moreover, rechallenge with a potential causative drug to establish a diagnosis is also one of the diagnostic methods in the RUCAM/ CIOMS criteria, but from an ethical point of view it is not recommended in children due to potential life-threatening adverse events.

The element combining diagnostics and therapy of drug toxicity is the discontinuation of treatment. Im- 
provement of laboratory parameters (normalisation of aminotransferases) confirms the adverse effect of the drug on the liver function. We found sustained normalisation of ALT activity 3 months after montelukast discontinuation.

\section{Conclusions}

The exclusion of other causes of increased activity of aminotransferases (viral, metabolic, autoimmune), improvement after dechallenge, the morphological findings and previous reports of comparable cases support the diagnosis of montelukast-induced liver injury in this boy. Physicians should strictly analyse indications for this drug and be aware of potential DILI caused by this agent. Therefore, the periodical assessment of aminotransferases should be recommended during treatment with this leukotriene modifier.

\section{References}

1. Fontana RJ, Seeff LB, Andrade RJ, et al. Standarization of nomenclature and causality assessment in drug-induced liver injury: summary of a clinical research workshop. Hepatology 2010; 52: 730-42.

2. Meier Y, Cavallaro M, Ross M, et al. Incidence of drug-induced liver injury in medical inpatients. Eur J Clin Pharmacol 2005; 61: 135-43.

3. Murray KF, Hadzic N, Wirth S, et al. Drug-related hepatotoxicity and acute liver failure. J Pediatr Gastroenterol Nutr 2008; 47: 395-405.

4. Russmann $\mathrm{S}$, Iselin $\mathrm{HU}$, Meier D, et al. Acute hepatitis associated with montelukast. J Hepatol 2003; 38: 694-5.

5. Sass DA, Chopra KB, Wu T. A case of montelukast-induced hepatotoxicity. Am J Gastroenterol 2003; 98: 704-5.

6. Margery J, Dot JM, Bredin C, et al. Montelukast induced cytolytic acute hepatitis. Gastroenterol Clin Biol 2003; 27: 129-30.

7. Goldstein MF, Anoia J, Black M. Montelukast-induced hepatitis. Ann Intern Med 2004; 140: 586-7.

8. Actis GC, Bugianesi E, Ottobrelli A, Rizzetto M. Fatal liver failure following food supplements during chronic treatment with montelukast. Dig Liver Dis 2007; 39: 953-5.

9. Harugeri A, Parthasarathi G, Sharma J, et al. Montelukast induced acute hepatocellular liver injury. J Postgrad Med 2009; 55: 141-2.

10. Incecik F, Onlen Y, Sangun O, Akoglu S. Probable montelukast-induced hepatotoxicity in a pediatric patient: case report. Ann Saudi Med 2007; 27: 462-3.

Received: 24.10.2011

Accepted: 27.03 .2012 